\title{
Weighted variable exponent grand Lebesgue spaces and inequalities of approximation
}

\author{
İsmail Aydın¹ (10, Ramazan Akgün² (1) \\ ${ }^{1}$ Sinop University, Faculty of Arts and Sciences, Department of Mathematics, Sinop, Turkey \\ ${ }^{2}$ Balikesir University, Faculty of Arts and Sciences, Department of Mathematics, Balikesir, Turkey
}

\begin{abstract}
In this paper we discuss and investigate trigonometric approximation in weighted grand variable exponent Lebesgue spaces. We also prove the direct and inverse theorems in these spaces.
\end{abstract}

Mathematics Subject Classification (2020). 46E35, 43A15, 46E30

Keywords. weighted grand variable exponent Lebesgue, Sobolev and Lipschitz space, maximal operator, modulus of smoothness, best approximation, Jackson and inverse theorems, K-functional

\section{Introduction}

In 1992, T. Iwaniec and C. Sbordone [22] introduced the grand Lebesgue spaces $L^{p)}(\Omega)$, $1<p<\infty$, on bounded sets $\Omega \subset \mathbb{R}^{d}$, with applications to differential equations. A generalized version $L^{p), \theta}(\Omega)$ appeared in L. Greco, T. Iwaniec and C. Sbordone [18]. During last years these spaces were intensively studied for various applications (see, e.g., $[1,16-18,20,22,23])$. The variable exponent Lebesgue spaces (or generalized Lebesgue spaces) $L^{p(.)}$ appeared in literature for the first time in 1931 with an article written by Orlicz [25]. Kováčik and Rákosník [24] introduced the variable exponent Lebesgue space $L^{p(.)}\left(\mathbb{R}^{d}\right)$ and Sobolev space $\mathcal{W}^{k, p(.)}\left(\mathbb{R}^{d}\right)$ in higher dimensional Euclidean spaces. There are several applications of these spaces, such as, elastic mechanics, electrorheological fluids, image restoration and nonlinear degenerated partial differential equations (see $[10,11,14])$. The spaces $L^{p(.)}\left(\mathbb{R}^{d}\right)$ and $L^{p}\left(\mathbb{R}^{d}\right)$ have many common properties, such as Banach space, reflexivity, separability, uniform convexity, Hölder inequalities and embeddings. A crucial difference between $L^{p(.)}\left(\mathbb{R}^{d}\right)$ and $L^{p}\left(\mathbb{R}^{d}\right)$ is that the variable exponent Lebesgue space is not invariant under translation in general, see [13, Lemma 2.3] and [24, Example 2.9]. For more information see $[10,14]$. The grand variable exponent Lebesgue space $L^{p(.), \theta}(\Omega)$ was introduced and studied by Kokilasvili and Meski [23]. In their studies they established the boundedness of maximal and Calderon operators in these spaces. The space $L^{p(.), \theta}(\Omega)$ is not reflexive, separable, rearrangement invariant and translation invariant. There are several published papers about direct and inverse theorems of approximation theory in some function spaces weighted, variable or non-weighted, see, [2-8, 12, 19, 21].

\footnotetext{
*Corresponding Author.

Email addresses: iaydin@sinop.edu.tr (İ. Aydın), rakgun@balikesir.edu.tr (R. Akgün)

Received: 03.02.2020; Accepted: 29.05.2020
} 
In this study we obtain some inequalities involving trigonometric polynomial approximation in a certain subspace of the weighted variable exponent grand Lebesgue space $L_{w}^{p(.), \theta}$. Also we give some basic properties of these spaces. Finally, we prove some direct and inverse theorems of approximation in $L_{w}^{p(.), \theta}$.

\section{Notations and preliminaries}

In this section, we give some essential definitions, theorems and remarks for weighted grand variable exponent Lebesgue spaces.

Definition 2.1. Let $\mathbb{T}:=[0,2 \pi]$ and let $p():. \mathbb{T} \longrightarrow[1, \infty)$ be a measurable $2 \pi$-periodic function such that

$$
1 \leq p^{-}=\underset{x \in \mathbb{T}}{\operatorname{ess} \inf } p(x) \leq \underset{x \in \mathbb{T}}{\operatorname{ess} \sup } p(x):=p^{+}<\infty .
$$

Assume that $p($.$) satisfies the local log-continuity condition, i.e., there exists a constant$ $C>0$ such that the inequality

$$
|p(x)-p(y)| \leq \frac{C}{-\log |x-y|}
$$

holds for all $x, y \in \mathbb{T}$ with $|x-y| \leq \frac{1}{2}$ (briefly $p(.) \in P(\mathbb{T})$ ). We also define a subclass

$$
P_{0}(\mathbb{T})=\left\{p(.) \in P(\mathbb{T}): 1<p^{-}\right\} .
$$

Definition 2.2. Let $p(.) \in P(\mathbb{T})$. Variable exponent Lebesgue space $L^{p(.)}:=L^{p(.)}(\mathbb{T})$ is defined as the set of all measurable, $2 \pi$-periodic functions $f$ on $\mathbb{T}$ such that $\varrho_{p(.)}(\lambda f)<\infty$ for some $\lambda>0$, equipped with the Luxemburg norm

$$
\|f\|_{p(.)}=\inf \left\{\lambda>0: \varrho_{p(.)}\left(\frac{f}{\lambda}\right) \leq 1\right\}
$$

where $\varrho_{p(.)}(f)=\int_{\mathbb{T}}|f(x)|^{p(x)} d x$. The space $L^{p(.)}$ is a Banach space with the norm $\|\cdot\|_{p(.)}$. Moreover, the norm $\|\cdot\|_{p(.)}$ coincides with the usual Lebesgue norm $\|\cdot\|_{p}$ whenever $p()=$. is a constant function. If $p^{+}<\infty$, then $f \in L^{p(.)}$ if and only if $\varrho_{p(.)}(f)<\infty$.

Definition 2.3. A Lebesgue measurable and locally integrable function $w: \mathbb{T} \longrightarrow(0, \infty)$ is called a weight function. Suppose that $p(.) \in P(\mathbb{T})$. The weighted modular is defined by

$$
\varrho_{p(.), w}(f)=\int_{\mathbb{T}}|f(x)|^{p(x)} w(x) d x .
$$

The weighted variable exponent Lebesgue space $L_{w}^{p(.)}:=L_{w}^{p(.)}(\mathbb{T})$ consists of all measurable functions $f$ on $\mathbb{T}$ for which $\|f\|_{p(.), w}=\left\|f w^{\frac{1}{p(.)}}\right\|_{p(.)}<\infty$. Also, $L_{w}^{p(.)}$ is a uniformly convex Banach space, thus reflexive.

Remark 2.4. Let $w$ be a weight on $\mathbb{T}$ and $p(.) \in P(\mathbb{T})$.

(i) Relations between the modular $\varrho_{p(.), w}($.$) and \|\cdot\|_{p(.), w}$ are as follows:

$$
\begin{gathered}
\min \left\{\varrho_{p(.), w}(f)^{\frac{1}{p^{-}}}, \varrho_{p(.), w}(f)^{\frac{1}{p^{+}}}\right\} \leq\|f\|_{p(.), w} \leq \max \left\{\varrho_{p(.), w}(f)^{\frac{1}{p^{-}}}, \varrho_{p(.), w}(f)^{\frac{1}{p^{+}}}\right\}, \\
\min \left\{\|f\|_{p(.), w}^{p^{+}},\|f\|_{p(.), w}^{p^{-}}\right\} \leq \varrho_{p(.), w}(f) \leq \max \left\{\|f\|_{p(.), w}^{p^{+}},\|f\|_{p(.), w}^{p^{-}}\right\} .
\end{gathered}
$$

(ii) If $0<C \leq w$, then we have $L_{w}^{p(.)} \hookrightarrow L^{p(.)}$, since one gets easily that

$$
C \int_{\mathbb{T}}|f(x)|^{p(x)} d x \leq \int_{\mathbb{T}}|f(x)|^{p(x)} w(x) d x
$$


and $C\|f\|_{p(.)} \leq\|f\|_{p(.), w}$ (see [9]). Moreover, due to $|\mathbb{T}|<\infty$ and $1 \leq p($.$) we have$ $L_{w}^{p(.)}(\mathbb{T}) \hookrightarrow L^{p(.)}(\mathbb{T}) \hookrightarrow L^{1}(\mathbb{T})$.

Definition 2.5. Let $\theta>0$ and $p(.) \in P(\mathbb{T})$. The grand variable exponent Lebesgue space, $L^{p(.), \theta}$, is the class of all measurable functions $f$ for which

$$
\|f\|_{p(.), \theta}:=\sup _{0<\varepsilon<p^{-}-1} \varepsilon^{\frac{\theta}{p^{-}-\varepsilon}}\|f\|_{p(.)-\varepsilon}<\infty .
$$

When $p()=$.$p is a constant function, these spaces coincide with the grand Lebesgue$ spaces $L^{p), \theta}(\mathbb{T})$.

Definition 2.6. Let $w$ be a weight on $\mathbb{T}$ and $p(.) \in P(\mathbb{T})$. The weighted grand variable exponent Lebesgue spaces $L_{w}^{p(.), \theta}:=L_{w}^{p(.), \theta}(\mathbb{T})$ is the class of all measurable functions $f$ for which

$$
\|f\|_{p(.), w, \theta}:=\sup _{0<\varepsilon<p^{-}-1} \varepsilon^{\frac{\theta}{p^{-}-\varepsilon}}\|f\|_{p(.)-\varepsilon, w}<\infty .
$$

Remark 2.7. Let $w$ be a weight on $\mathbb{T}$ and $p(.) \in P(\mathbb{T})$.

(i) It is easy to see that the following continuous embeddings hold

$$
L^{p(.)} \hookrightarrow L^{p(\cdot), \theta} \hookrightarrow L^{p(.)-\varepsilon} \hookrightarrow L^{1}, 0<\varepsilon<p^{-}-1
$$

due to $|\mathbb{T}|<\infty$ (see $[12,23])$.

(ii) For $f \in L_{w}^{p(.), \theta}(\mathbb{T})$ the norm equality $\|f\|_{p(.), w, \theta}=\left\|f w^{\frac{1}{p(.)}}\right\|_{p(.), \theta}$ is not valid in $L_{w}^{p(.), \theta}(\mathbb{T})($ see $[17])$.

Example 2.8. Let $\alpha>0, \theta=1, p()=p=$. constant and choose a weight $w(x)=x^{\alpha}$. If we take $f(x)=x^{\beta}$ for $\beta>-\alpha-1$, then we have $f \in L_{w}^{p)}(0,1)$. But, $\left(f w^{\frac{1}{p}}\right)^{p-\varepsilon}$ is not integrable in $(0,1)$ for any $0<\varepsilon<p-1$ and so $f w^{\frac{1}{p}} \notin L^{p)}(0,1)$ (see [16]).

Proposition 2.9 (Nesting Property). If $0<C \leq w, p(.) \in \mathrm{P}(\mathbb{T})$ and $\theta_{1}<\theta_{2}$, then we have the following continuous embeddings

$$
L_{w}^{p(.)} \hookrightarrow L_{w}^{p(.), \theta_{1}} \hookrightarrow L_{w}^{p(.), \theta_{2}} \hookrightarrow L_{w}^{p(.)-\varepsilon} \hookrightarrow L^{p(.)-\varepsilon} \hookrightarrow L^{1}, 0<\varepsilon<p^{-}-1
$$

due to $|\mathbb{T}|<\infty$ (see $[12,23])$.

Remark 2.10. Let $w$ be a weight on $\mathbb{T}$ and $p(.) \in P(\mathbb{T})$. There are several differences between $L_{w}^{p(.)}$ and $L_{w}^{p(.), \theta}$. For instance, the set of the bounded functions is not dense in $L_{w}^{p(.), \theta}$, and the closure of $L^{\infty}(\mathbb{T})$ in the norm of $L_{w}^{p(.), \theta}$ can be characterized by the functions $f$ such that

$$
\lim _{\varepsilon \rightarrow 0} \sup \varepsilon^{\frac{\theta}{p^{-}-\varepsilon}}\|f\|_{p(.)-\varepsilon, w}=0
$$

(see [1]). Moreover, the closure of simple functions is not dense in $L_{w}^{p(.), \theta}$. Also, the space $L_{w}^{p(.), \theta}$ is not reflexive, not separable and not rearrangement invariant. Since the closure of $L_{w}^{p(.)}$ in $L_{w}^{p(.), \theta}$ does not coincide with the latter space, that is, $L_{w}^{p(.)}$ is not dense in $L_{w}^{p(.), \theta}$, then we redefine this set in the following theorem as a subspace of $L_{w}^{p(.), \theta}$ (see [12,23]).

Theorem 2.11. Let $w$ be a weight on $\mathbb{T}$ and $p(.) \in \mathrm{P}(\mathbb{T})$. The following statements hold:

(i) The space $L_{w}^{p(.), \theta}$ is complete.

(ii) The closure of $L_{w}^{p(.)}$ in $L_{w}^{p(.), \theta}$ consists of functions $f$, which belong to $L_{w}^{p(.), \theta}$, for which $\lim _{\varepsilon \rightarrow 0} \varepsilon^{\frac{\theta}{p^{-}-\varepsilon}}\|f\|_{p(.)-\varepsilon, w}=0$. 
Proof. (i) Let $\left(f_{n}\right)_{n \in \mathbb{N}}$ be a Cauchy sequence in $L_{w}^{p(.), \theta}$. Then for all $\eta>0$ there exists $N(\eta)>0$ such that, whenever $n, m>N(\eta)$ we have

$$
\varepsilon^{\frac{\theta}{p^{-}-\varepsilon}}\left\|f_{n}-f_{m}\right\|_{p(.)-\varepsilon, w}<\frac{\eta}{3}
$$

for any $\varepsilon \in\left(0, p^{-}-1\right)$. Therefore $\left(f_{n}\right)_{n \in \mathbb{N}}$ is a Cauchy sequence in $L_{w}^{p(.)-\varepsilon}$ for arbitrary $\varepsilon \in\left(0, p^{-}-1\right)$. Then there is an $f$ in $L_{w}^{p(.)}-\varepsilon$ such that

$$
\left\|f-f_{n}\right\|_{p(.)-\varepsilon, w} \rightarrow 0
$$

for every $\varepsilon \in\left(0, p^{-}-1\right)$ (note that the function $f$ is unique for all $\varepsilon \in\left(0, p^{-}-1\right)$, see [23]). For $n>N(\eta)$, there is an $\varepsilon_{0}(n) \in\left(0, p^{-}-1\right)$ such that

$$
\left\|f-f_{n}\right\|_{p(.), w, \theta} \leq \varepsilon_{0}(n)^{\frac{\theta}{p^{-}-\varepsilon}}\left\|f-f_{n}\right\|_{p(.)-\varepsilon_{0}(n), w}+\frac{\eta}{3}
$$

by using the definition of the supremum. Moreover, there exists $N_{1} \in \mathbb{N}$ such that for $m>N_{1}$ we have

$$
\varepsilon^{\frac{\theta}{p^{-}-\varepsilon_{0}(n)}}\left\|f-f_{m}\right\|_{p(.)-\varepsilon_{0}(n), w} \leq \frac{\eta}{3}
$$

due to (2.2). If we combine (2.3), (2.4) and (2.1), then we get

$$
\begin{gathered}
\left\|f-f_{n}\right\|_{p(.), w, \theta} \leq \varepsilon_{0}(n)^{\frac{\theta}{p^{-}-\varepsilon}}\left\|f-f_{n}\right\|_{p(.)-\varepsilon_{0}(n), w}+\frac{\eta}{3} \\
\leq \varepsilon_{0}(n)^{\frac{\theta}{p^{-}-\varepsilon}}\left\|f_{n}-f_{m}\right\|_{p(.)-\varepsilon_{0}(n), w}+\varepsilon_{0}(n)^{\frac{\theta}{p^{-}-\varepsilon}}\left\|f-f_{m}\right\|_{p(.)-\varepsilon_{0}(n), w}+\frac{\eta}{3} \\
\leq \frac{\eta}{3}+\frac{\eta}{3}+\frac{\eta}{3}=\eta
\end{gathered}
$$

for $n>N(\eta)$ and $m>N_{1}$. This completes the proof of (i).

(ii) Denote by $\left[L_{w}^{p(.)}\right]_{p(.), w, \theta}$ the closure of $L_{w}^{p(.)}$ in $L_{w}^{p(.), \theta}$. For $f \in\left[L_{w}^{p(.)}\right]_{p(.), w, \theta}$ we can obtain that there is a sequence $\left(f_{n}\right)_{n \in \mathbb{N}}$ in $L_{w}^{p(.)}$ such that $\left\|f-f_{n}\right\|_{p(.), w, \theta} \rightarrow 0$ by the definition of the closure set. Then, for fixed $\delta>0$, there exists $N=N(\delta)>0$ such that, whenever $n>N(\delta)$ we obtain

$$
\left\|f-f_{n}\right\|_{p(.), w, \theta}<\frac{\delta}{2}
$$

It is well-known that the continuous embedding $L_{w}^{q(.)}(\mathbb{T}) \hookrightarrow L_{w}^{p(.)}(\mathbb{T})$ holds if and only if $q(.) \geq p($.$) because of |\mathbb{T}|<\infty[24]$. Hence we get

$$
\varepsilon^{\frac{\theta}{p^{-}-\varepsilon}}\left\|f_{n}\right\|_{p(.)-\varepsilon, w} \leq(1+|\mathbb{T}|) \varepsilon^{\frac{\theta}{p^{-}-\varepsilon}}\left\|f_{n}\right\|_{p(.), w} \rightarrow 0
$$

as $\varepsilon \rightarrow 0$. If we take $\varepsilon_{0}>0$ such that $0<\varepsilon<\varepsilon_{0}$, then we can write

$$
\frac{\theta}{\varepsilon^{\frac{\theta}{p^{-}-\varepsilon}}}\left\|f_{n}\right\|_{p(.)-\varepsilon, w}<\frac{\delta}{2} \text {. }
$$

Finally, if we collect (2.5) and (2.7), then we have

$$
\begin{aligned}
\varepsilon^{\frac{\theta}{p^{-}-\varepsilon}}\|f\|_{p(.)-\varepsilon, w} & \leq \varepsilon^{\frac{\theta}{p^{-}-\varepsilon}}\left\|f-f_{n}\right\|_{p(.)-\varepsilon, w}+\varepsilon^{\frac{\theta}{p^{-}-\varepsilon}}\left\|f_{n}\right\|_{p(.)-\varepsilon, w} \\
& \leq\left\|f-f_{n}\right\|_{p(.), w, \theta}+\frac{\delta}{2} \leq \delta
\end{aligned}
$$

as $\varepsilon \rightarrow 0$. 
Definition 2.12. We denote the closure of $L_{w}^{p(.)}$ by $L_{0, w}^{p(.), \theta}$. For $f \in L_{0, w}^{p(.), \theta}(\mathbb{T})$ we have

$$
\lim _{\varepsilon \rightarrow 0} \varepsilon^{\frac{\theta}{p^{-}-\varepsilon}}\|f\|_{p(.)-\varepsilon, w}=0
$$

by the last theorem (see [12]).

Proposition 2.13. Let $w$ be a weight on $\mathbb{T}$ and $p(.) \in \mathrm{P}(\mathbb{T})$. Then, $\left(L_{w}^{p(.), \theta}(\mathbb{T}),\|\cdot\|_{p(.), w, \theta}\right)$ is a Banach function space (see [1]).

We denote the Hardy-Littlewood maximal operator $M f$ of $f$ by

$$
M f(x)=\sup _{I} \frac{1}{|I|} \int_{I}|f(t)| d t, \quad t \in \mathbb{T},
$$

where the supremum is taken over all intervals $I$ whose length is less than $2 \pi$.

The boundedness of the Hardy-Littlewood maximal operator $M$ on the space $L_{W}^{p(.), \theta}$, $\theta>0, p(.) \in P_{0}(\mathbb{T})$, was proved in the following theorem for power weights of the form $W(x)=\left|x-x_{0}\right|^{\gamma}$, where $x_{0} \in \mathbb{T},-1<\gamma<p\left(x_{0}\right)-1$.

Theorem 2.14. ([17]) Let $p(.) \in \mathrm{P}_{0}(\mathbb{T}), x_{0} \in(-\pi, \pi), \theta>0$, and $-1<\gamma<p\left(x_{0}\right)-1$. Then the operator $M$ is bounded in $L_{W}^{p(.), \theta}$, i.e. for all $f \in L_{W}^{p(.), \theta}$ there exists a $C>0$ such that the inequality

holds with $W(x)=\left|x-x_{0}\right|^{\gamma}$.

$$
\|M f\|_{p(.), W, \theta} \leq C\|f\|_{p(.), W, \theta}
$$

In what follows, all weights $W$ considered will be power weight of the form $W(x)=$ $\left|x-x_{0}\right|^{\gamma}$ satisfying the hypothesis of the last theorem.

Since $W(x)=\left|x-x_{0}\right|^{\gamma}$ satisfies the $A_{p(.)}$ condition of Muckenhoupt weights, then we have the continuous embedding $L_{W}^{p(.), \theta} \hookrightarrow L^{1}(\mathbb{T})[8]$. This means that we can consider the corresponding Fourier series of $f \in L_{W}^{p(.), \theta}$ given by

$$
f(x) \sim \frac{a_{0}(f)}{2}+\sum_{k=1}^{\infty}\left(a_{k}(f) \cos k x+b_{k}(f) \sin k x\right),
$$

where $a_{0}(f)=\pi^{-1} \int_{\mathbb{T}} f(t) d t$ and

$$
a_{k}(f)=\pi^{-1} \int_{\mathbb{T}} f(t) \cos k t d t, \quad b_{k}(f)=\pi^{-1} \int_{\mathbb{T}} f(t) \sin k t d t, \quad k=1,2, \ldots
$$

The $n$-th partial sums of the series (2.8) is defined by

$$
S_{n}(x, f):=\sum_{k=0}^{n} A_{k}(f)(x)=\frac{a_{0}(f)}{2}+\sum_{k=1}^{n}\left(a_{k}(f) \cos k x+b_{k}(f) \sin k x\right) .
$$

Definition 2.15. Let $W(x)=\left|x-x_{0}\right|^{\gamma}, \theta>0, p(.) \in P_{0}(\mathbb{T}), r=1,2, \ldots$ and $f \in L_{0, W}^{p(.), \theta}$. Then the $r$-th modulus of smoothness $\Omega_{r}(f, .)_{p(.), W, \theta}:[0, \infty) \rightarrow[0, \infty)$ is defined as

$$
\Omega_{r}(f, \delta)_{p(.), W, \theta}=\sup _{0<h \leq \delta}\left\|\rho_{h}^{r} f\right\|_{p(.), W, \theta}, r \in \mathbb{N},
$$

where

$$
\begin{gathered}
\rho_{h}^{r} f(x):=\frac{1}{h} \int_{0}^{h} \triangle_{t}^{r} f(x) d t, \\
\triangle_{t}^{r} f(x):=\sum_{s=0}^{r}(-1)^{r+s+1} b_{r, s} f(x+s t), \quad t>0,
\end{gathered}
$$

and $b_{r, s}$ are binomial coefficients. 
Remark 2.16. Using Theorem 2.14 we get

$$
\sup _{0<h \leq \delta}\left\|\rho_{h}^{r} f\right\|_{p(.), W, \theta} \leq C\|f\|_{p(.), W, \theta}<\infty .
$$

This shows that the function $\Omega_{r}(f, \delta)_{p(.), W, \theta}$ is well defined.

Remark 2.17. The modulus of smoothness $\Omega_{r}(f, \delta)_{p(.), W, \theta}$ has the following properties:

(i) $\Omega_{r}(f, \delta)_{p(.), W, \theta}$ is a non-negative, non-decreasing function of $\delta>0$.

(ii) $\Omega_{r}\left(f_{1}+f_{2}, .\right)_{p(.), W, \theta} \leq \Omega_{r}\left(f_{1}, .\right)_{p(.), W, \theta}+\Omega_{r}\left(f_{2}, .\right)_{p(.), W, \theta}$.

(iii) $\lim _{\delta \rightarrow 0} \Omega_{r}(f, \delta)_{p(.), W, \theta}=0$.

Definition 2.18. The best approximation error $E_{n}(f)_{p(.), W, \theta}$ of $f \in L_{0, W}^{p(.), \theta}$ is defined by

$$
E_{n}(f)_{p(.), W, \theta}:=\inf \left\{\left\|f-T_{n}\right\|_{p(.), W, \theta}: T_{n} \in \Pi_{n}\right\}
$$

where $\Pi_{n}$ is the set of trigonometric polynomials of degree at most $n$.

Definition 2.19. The Sobolev space $\mathcal{W}_{p(.), W, \theta}^{r}$ is the class of functions $f \in L_{W}^{p(.), \theta}$ such that $f^{(r)} \in L_{W}^{p(.), \theta}$ and

$$
\|f\|_{p(.), W, \theta}^{r}=\|f\|_{p(.), W, \theta}+\left\|f^{(r)}\right\|_{p(.), W, \theta}<\infty,
$$

for $r=1,2, \ldots$. Also the space $\mathcal{W}_{p(.), W, \theta}^{r}$ is a Banach space with respect to $\|\cdot\|_{p(.), W, \theta}^{r}$. We define

$$
\mathcal{W}_{0, p(.), W, \theta}^{r}=\left\{f: f \in L_{0, W}^{p(.), \theta} \cap \mathcal{W}_{p(.), W, \theta}^{r}\right\}
$$

\section{Main results}

The main results of this paper are the following theorems.

Theorem 3.1. Let $W(x)=\left|x-x_{0}\right|^{\gamma}, \theta>0, p(.) \in \mathrm{P}_{0}(\mathbb{T})$ and $r, n \in \mathbb{N}$. If $f \in \mathcal{W}_{0, p(.), W, \theta}^{r}$, then

$$
E_{n}(f)_{p(.), W, \theta} \leq \frac{c}{n^{r}} E_{n}\left(f^{(r)}\right)_{p(.), W, \theta}
$$

with a constant $c>0$ independent of $n$.

Corollary 3.2. Under the conditions of Theorem 3.1,

$$
E_{n}(f)_{p(.), W, \theta} \leq \frac{c}{n^{r}}\left\|f^{(r)}\right\|_{p(.), W, \theta}
$$

with a constant $c>0$ independent of $n=0,1,2,3, \ldots$.

Theorem 3.3. Let $W(x)=\left|x-x_{0}\right|^{\gamma}, \theta>0, p(.) \in \mathrm{P}_{0}(\mathbb{T})$ and $r, n \in \mathbb{N}$. If $f \in L_{0, W}^{p(.), \theta}$, then

$$
E_{n}(f)_{p(.), W, \theta} \leq c \Omega_{r}\left(f, \frac{1}{n}\right)_{p(.), W, \theta}
$$

with a constant $c>0$ independent of $n$.

Theorem 3.4. Let $W(x)=\left|x-x_{0}\right|^{\gamma}, \theta>0, p(.) \in \mathrm{P}_{0}(\mathbb{T})$ and $r, n \in \mathbb{N}$. If $f \in L_{0, W}^{p(.), \theta}$, then

$$
\Omega_{r}\left(f, \frac{1}{n}\right)_{p(.), W, \theta} \leq \frac{c}{n^{r}} \sum_{k=0}^{n}(k+1)^{r-1} E_{k}(f)_{p(.), W, \theta}
$$

with a constant $c>0$ independent of $n$.

To prove main results we need some lemmas and propositions given below. 
Lemma 3.5. Let $W(x)=\left|x-x_{0}\right|^{\gamma}, \theta>0, p(.) \in \mathrm{P}_{0}(\mathbb{T})$ and $r \in \mathbb{N}$. If $f \in \mathcal{W}_{0, p(.), W, \theta}^{r}$, then

$$
\Omega_{r}(f, \delta)_{p(.), W, \theta} \leq c \delta^{r}\left\|f^{(r)}\right\|_{p(.), W, \theta}
$$

with a constant $c>0$ independent of $n$.

Proof. Since

$$
\triangle_{t}^{r} f(.)=\int_{0}^{t} \int_{0}^{t} \ldots \int_{0}^{t} f^{(r)}\left(.+t_{1}+\ldots+t_{r}\right) d t_{1} \ldots d t_{r},
$$

applying ( $r$ times) the generalized Minkowski's inequality we get

$$
\begin{aligned}
& \qquad \frac{1}{h} \int_{0}^{h} \triangle_{t}^{r} f d t\left\|_{p(.), W, \theta} \leq \frac{c_{1}(p)}{h} \int_{0}^{h}\right\| \triangle_{t}^{r} f \|_{p(.), W, \theta} d t \\
& \leq h^{r} \frac{c_{1}(p)}{h^{r+1}} \int_{0}^{h}\left\|\int_{0}^{t} \ldots \int_{0}^{t} f^{(r)}\left(.+t_{1}+\ldots+t_{r}\right) d t_{1} \ldots d t_{r}\right\|_{p(.), W, \theta} d t \\
& =h^{r} \frac{c_{1}(p)}{h} \int_{0}^{h}\left\|\frac{1}{h} \int_{0}^{t}\left|\frac{1}{h^{r-1}} \int_{0}^{t} \ldots \int_{0}^{t} f^{(r)}\left(.+t_{1}+\ldots+t_{r}\right) d t_{1} \ldots d t_{r-1}\right|_{p} d t_{r}\right\|_{p(.), W, \theta} d t \\
& \leq h^{r} \frac{c_{2}(p)}{h} \int_{0}^{h}\left\|\frac{1}{h^{r-1}} \int_{0}^{t} \ldots \int_{0}^{t} f^{(r)}\left(.+t_{1}+\ldots+t_{r-1}\right) d t_{1} \ldots d t_{r-1}\right\|_{p(.), W, \theta} d t \\
& \left.\leq \ldots \leq h^{r} \frac{c_{3}(p, r)}{h} \int_{0}^{h} \| \int_{0} \frac{1}{h} \int_{0}^{h} f^{(r)}\left(.+t_{1}\right) d t_{1}\right\} \|_{p(.), W, \theta} d t \\
& \leq c_{4}(p, r) h^{r}\left\|f^{(r)}\right\|_{p(.), W, \theta} \frac{1}{h} \int_{0}^{h} d t=c_{4}(p, r) h^{r}\left\|f^{(r)}\right\|_{p(.), W, \theta},
\end{aligned}
$$

and taking supremum on $0<h \leq \delta$, we obtain the required inequality

$$
\Omega_{r}(f, \delta)_{p(.), W, \theta} \leq c \delta^{r}\left\|f^{(r)}\right\|_{p(.), W, \theta} .
$$

Definition 3.6. Let $W(x)=\left|x-x_{0}\right|^{\gamma}, \theta>0, p(.) \in P_{0}(\mathbb{T}), r \in \mathbb{N}$ and $f \in L_{0, W}^{p(.), \theta}$. We define Peetre's $K$-functional as

$$
K_{r}(f, \delta)_{p(.), W, \theta}:=\inf \left\{\|f-g\|_{p(.), W, \theta}+\delta^{r}\left\|g^{(r)}\right\|_{p(.), W, \theta}: g \in \mathcal{W}_{0, p(.), W, \theta}^{r}, \delta>0\right\} .
$$

Theorem 3.7. Let $W(x)=\left|x-x_{0}\right|^{\gamma}, \theta>0, p(.) \in \mathrm{P}_{0}(\mathbb{T}), r \in \mathbb{N}$. If $f \in L_{0, W}^{p(.), \theta}$, then there are some constants $c_{6}, c_{7}>0$ independent of $\delta$ such that

$$
c_{6} \Omega_{r}(f, \delta)_{p(.), W, \theta} \leq K_{r}(f, \delta)_{p(.), W, \theta} \leq c_{7} \Omega_{r}(f, \delta)_{p(.), W, \theta} .
$$

Proof. Let $f \in L_{0, W}^{p(.), \theta}$ and $g \in \mathcal{W}_{0, p(.), W, \theta}^{r}$. By Lemma 3.5 and Remark 2.17,

$$
\begin{aligned}
\Omega_{r}(f, \delta)_{p(.), W, \theta} & \leq \Omega_{r}(f-g, \delta)_{p(.), W, \theta}+\Omega_{r}(g, \delta)_{p(.), W, \theta} \\
& \leq c\left(\|f-g\|_{p(.), W, \theta}+\delta^{r}\left\|g^{(r)}\right\|_{p(.), W, \theta}\right)
\end{aligned}
$$


and taking infimum with respect to $g \in \mathcal{W}_{0, p(.), W, \theta}^{r}$ in the last inequality we have

$$
\Omega_{r}(f, \delta)_{p(.), W, \theta} \leq c K_{r}(f, \delta)_{p(.), W, \theta} \cdot
$$

In order to prove the reverse of the last inequality we define the function

$$
f_{r, \delta}(x)=\frac{2}{\delta} \int_{\frac{\delta}{2}}^{\delta}\left(\frac{1}{h^{r}} \sum_{s=0}^{r-1}(-1)^{r+s+1}\left(\begin{array}{l}
r \\
s
\end{array}\right) \int_{0}^{h} \ldots \int_{0}^{h} f\left(x+\frac{r-s}{r}\left[t_{1}+\ldots+t_{r}\right]\right) d t_{1} \ldots d t_{r}\right) d h
$$

for $\delta>0$ and $r \geq 1$. Then, differentiating $r-1$ times and setting $t:=\frac{r-s}{r} t_{r}$ we see that

$$
\begin{aligned}
& \left\{\int_{0}^{h} \ldots \int_{0}^{h} f\left(x+\frac{r-s}{r}\left[t_{1}+\ldots+t_{r}\right]\right) d t_{1} \ldots d t_{r}\right\}^{(r-1)} \\
= & \left\{\int_{0}^{h}\left(\frac{r}{r-s}\right)^{r-1} \sum_{m=0}^{r-1}\left(\begin{array}{c}
r-1 \\
m
\end{array}\right)(-1)^{r+m} f\left(x+\frac{r-s}{r} t_{r}+m \frac{r-s}{r} h\right) d t_{r}\right\} \\
= & \int_{0}^{h}\left(\frac{r}{r-s}\right)^{r-1} \triangle_{\frac{r-s}{r} h}^{r-1} f(x+t) d t,
\end{aligned}
$$

and then by $(3.1)$

$$
f_{r, \delta}^{(r-1)}(x):=\frac{2}{\delta} \int_{\frac{\delta}{2}}^{\delta} \frac{1}{h^{r}}\left\{\sum_{s=0}^{r-1} \int_{x}^{x+\frac{r-s}{r} h}(-1)^{r+s+1}\left(\begin{array}{l}
r \\
s
\end{array}\right) \triangle_{\frac{r-s}{r} h}^{r-1} f(t) d t\right\} d h .
$$

Now we prove $f_{r, \delta}^{(r)} \in L_{0, W}^{p(.), \theta}$. Differentiating the relation (3.2) we obtain

$$
f_{r, \delta}^{(r)}(x):=\frac{2}{\delta} \int_{\frac{\delta}{2}}^{\delta} \frac{1}{h^{r}}\left\{\sum_{s=0}^{r-1}(-1)^{r+s+1}\left(\begin{array}{l}
r \\
s
\end{array}\right)\left(\frac{r}{r-s}\right)^{r} \triangle_{\frac{r-s}{r} h}^{r} f(x)\right\} d h
$$

and denoting $t:=\frac{r-s}{r} h$ we have

$$
\begin{aligned}
&\left|f_{r, \delta}^{(r)}(x)\right| \leq \frac{2^{r+1}}{\delta^{r}} \sum_{s=0}^{r-1}\left(\begin{array}{l}
r \\
s
\end{array}\right)\left(\frac{r}{r-s}\right)^{r}\left|\frac{1}{\delta} \int_{\frac{\delta}{2}}^{\delta} \triangle_{\frac{r-s}{r} h}^{r} f(x) d h\right| \\
&=\frac{2^{r+1}}{\delta^{r}} \sum_{s=0}^{r-1}\left(\begin{array}{l}
r \\
s
\end{array}\right)\left(\frac{r}{r-s}\right)^{r}\left|\frac{1}{\frac{r-s}{r} \delta} \int_{\frac{r-s}{r}\left(\frac{\delta}{2}\right)}^{\frac{r-s}{r} \delta} \triangle_{t}^{r} f(x) d t\right| \\
& \leq \frac{2^{r+1}}{\delta^{r}} \sum_{s=0}^{r-1}\left(\begin{array}{l}
r \\
s
\end{array}\right)\left(\frac{r}{r-s}\right)^{r}\left\{\left|\frac{1}{\frac{r-s}{r} \delta} \int_{0}^{\frac{r-s}{r} \delta} \triangle_{t}^{r} f(x) d t\right|+\left|\frac{1}{\frac{r-s}{r} \delta} \int_{0}^{\frac{r-s}{r}\left(\frac{\delta}{2}\right)} \triangle_{t}^{r} f(x) d t\right|\right\},
\end{aligned}
$$

which implies the inequality

$$
\left\|f_{r, \delta}^{(r)}\right\|_{p(.), W, \theta} \leq 2 c(r) \delta^{-r} \Omega_{r}(f, \delta)_{p(.), W, \theta} \leq c_{5}(p, r)\|f\|_{p(.), W, \theta} \cdot
$$

Since $f \in L_{0, W}^{p(.), \theta}$, then $f_{r, \delta}^{(r)} \in L_{0, W}^{p(.), \theta}$. 
Let $f \in L_{0, W}^{p(.), \theta}$. For $\delta>0$ and $r=1,2, \ldots$, we have

$$
\left|f_{r, \delta}(x)-f(x)\right|=\left|\frac{2}{\delta} \int_{\frac{\delta}{2}}^{\delta}\left\{\frac{1}{h^{r}} \int_{0}^{h} \ldots \int_{0}^{h} \frac{\triangle_{\frac{t_{1}+\ldots+t_{r}}{r}}^{r}}{r}(x) d t_{1} \ldots d t_{r}\right\} d h\right|
$$

and by the generalized Minkowski's inequality

$$
\begin{aligned}
\| f_{r, \delta} & -f \|_{p(.), W, \theta} \leq c_{6}(p, r) \frac{2}{\delta} \int_{\frac{\delta}{2}}^{\delta}\left\{\frac{1}{h^{r-1}} \int_{0}^{h} \ldots \int_{0}^{h}\left\|\frac{1}{h} \int_{0}^{h} \triangle_{\frac{t_{1}+\ldots+t_{r}}{r}}^{r} f d t_{1}\right\|_{p(.), W, \theta} d t_{2} \ldots d t_{r}\right\} d h \\
& =c_{6}(p, r) \frac{2}{\delta} \int_{\frac{\delta}{2}}^{\delta}\left\{\frac{1}{h^{r-1}} \int_{0}^{h} \ldots \int_{0}^{h}\left\|\frac{1}{h} \int_{t_{2}+\ldots+t_{r}}^{h+t_{2}+\ldots+t_{r}} \triangle_{\frac{t}{r}}^{r} f d t\right\|_{p(.), W, \theta} d t_{2} \ldots d t_{r}\right\} d h .
\end{aligned}
$$

Since

$$
\begin{aligned}
& \left\|\frac{1}{h} \int_{t_{2}+\ldots+t_{r}}^{h+t_{2}+\ldots+t_{r}} \triangle_{\frac{t}{r}}^{r} f d t\right\|_{p(.), W, \theta}=\left\|\frac{1}{h}\left(\int_{0}^{h+t_{2}+\ldots+t_{r}} \triangle_{\frac{t}{r}}^{r} f d t-\int_{0}^{t_{2}+\ldots+t_{r}} \triangle_{\frac{t}{r}}^{r} f d t\right)\right\|_{p(.), W, \theta} \\
& \leq\left\|\frac{1}{\left(h+t_{2}+\ldots+t_{r}\right) / r} \int_{0}^{\left(h+t_{2}+\ldots+t_{r}\right) / r} \triangle_{\frac{t}{r}}^{r} f d t\right\|_{p(.), W, \theta} \\
& +\left\|\frac{1}{\left(t_{2}+\ldots+t_{r}\right) / r} \int_{0}^{\left(t_{2}+\ldots+t_{r}\right) / r} \triangle_{\frac{t}{r}}^{r} f d t\right\|_{p(.), W, \theta} \\
& =\sup _{\left(h+t_{2}+\ldots+t_{r}\right) / r \leq \delta}\left\|\frac{1}{\left(h+t_{2}+\ldots+t_{r}\right) / r} \int_{0}^{\left(h+t_{2}+\ldots+t_{r}\right) / r} \triangle_{\frac{t}{r}}^{r} f d t\right\|_{p(.), W, \theta} \\
& +\sup _{\left(t_{2}+\ldots+t_{r}\right) / r \leq \delta}\left\|\frac{1}{\left(t_{2}+\ldots+t_{r}\right) / r} \int_{0}^{\left(t_{2}+\ldots+t_{r}\right) / r} \triangle_{\frac{t}{r}}^{r} f d t\right\|_{p(.), W, \theta} \\
& =\Omega_{r}(f, \delta)_{p(.), W, \theta}+\Omega_{r}(f, \delta)_{p(.), W, \theta}=2 \Omega_{r}(f, \delta)_{p(.), W, \theta},
\end{aligned}
$$

then combining (3.4) and (3.5) we have

$$
\begin{aligned}
\left\|f_{r, \delta}-f\right\|_{p(.), W, \theta} & \leq c(p, r) \frac{2}{\delta} \int_{\frac{\delta}{2}}^{\delta}\left\{\frac{1}{h^{r-1}} \int_{0}^{h} \ldots \int_{0}^{h} \Omega_{r}(f, \delta)_{p(.), W, \theta} d t_{2} \ldots d t_{r}\right\} d h \\
& \leq c(p, r) \Omega_{r}(f, \delta)_{p(.), W, \theta} \frac{2}{\delta} \int_{\frac{\delta}{2}}^{\delta} d h=c(p, r) \Omega_{r}(f, \delta)_{p(.), W, \theta}
\end{aligned}
$$

Finally, if we use (3.3) and (3.6), then we get

$$
\begin{aligned}
K_{r}(f, \delta)_{p(.), W, \theta} & \leq\left\|f_{r, \delta}-f\right\|_{p(.), W, \theta}+\delta^{r}\left\|f_{r, \delta}^{(r)}\right\|_{p(.), W, \theta} \\
& \leq c_{7} \Omega_{r}(f, \delta)_{p(.), W, \theta} .
\end{aligned}
$$

This completes the proof.

The following lemma is a Bernstein inequality for $L_{W}^{p(.), \theta}$. 
Lemma 3.8. Let $W(x)=\left|x-x_{0}\right|^{\gamma}, \theta>0, p(.) \in \mathrm{P}_{0}(\mathbb{T}), r \in \mathbb{N}$. If $T_{n}$ is a trigonometric polynomial of degree at most $n$, then

$$
\left\|T_{n}^{\prime}\right\|_{p(.), W, \theta} \leq c n\left\|T_{n}\right\|_{p(.), W, \theta} .
$$

Proof. It is well-known that

$$
\sup _{n}\left|\sigma_{n}(x, f)\right| \leq c M f(x)
$$

with a constant $c>0$ independent of $f$ and $x \in \mathbb{T}$, where $\sigma_{n}(x, f)$ is the Cesàro means for a function $f \in L_{W}^{p(.), \theta}$ [27]. Using Theorem 2.14 we have

$$
\left\|\sup _{n}\left|\sigma_{n}(., f)\right|\right\|_{p(.), W, \theta} \leq c\|f\|_{p(.), W, \theta} .
$$

Since

$$
T_{n}(x)=\frac{1}{\pi} \int_{T} T_{n}(t) D_{n}(t-x) d t, \text { with } D_{n}(t)=\frac{1}{2}+\sum_{j=1}^{n} \cos j t,
$$

it is well-known that

$$
T_{n}^{\prime}(x)=2 n \sigma_{n-1}\left(x, T_{n}\right)
$$

and, hence,

$$
\left\|T_{n}^{\prime}\right\|_{p(.), W, \theta} \leq 2 n\left\|\sigma_{n-1}\left(.,\left|T_{n}\right|\right)\right\|_{p(.), W, \theta} \leq 2 c n\left\|T_{n}\right\|_{p(.), W, \theta} .
$$

This completes the proof.

Lemma 3.8 can be generalized for $r$-th derivative of $T_{n}$. For this we need a Minkowski's inequality for integrals. The following results were proved, when $W \equiv 1$, by Danelia and Kokilashvili [12, Proposition 2.4]. The same proof also suits our case below.

Lemma 3.9. Let $W(x)=\left|x-x_{0}\right|^{\gamma}, \theta>0, p(.) \in \mathrm{P}_{0}(\mathbb{T})$, and $f \in L_{0, W}^{p(.), \theta}$. If $f(x, y) a$ measurable function on $\mathbb{T} \times \mathbb{T}$, then, the following integral inequality holds

$$
\left\|\int_{\mathbb{T}} f(., y) d y\right\|_{p(.), W, \theta} \leq C \int_{\mathbb{T}}\|f(., y)\|_{p(.), W, \theta} d y .
$$

As a corollary of the last two lemmas we get:

Corollary 3.10. Let $W(x)=\left|x-x_{0}\right|^{\gamma}, \theta>0, p(.) \in \mathrm{P}_{0}(\mathbb{T})$ and $r \in \mathbb{N}$. If $T_{n}$ is a trigonometric polynomial of degree at most $n$, then

$$
\left\|T_{n}^{(r)}\right\|_{p(.), W, \theta} \leq c n^{r}\left\|T_{n}\right\|_{p(.), W, \theta} .
$$

\section{Proof of main results}

Let $n \in \mathbb{N}$ and

$$
D_{n} f(x):=\frac{1}{\pi} \int_{\mathbb{T}} f(x-t) J_{2,\left\lfloor\frac{n}{2}\right\rfloor+1}(t) d t
$$

be the Jackson operator (polynomial), where $\left\lfloor\frac{n}{2}\right\rfloor$ denotes the integer part of a real number $\frac{n}{2}$, and $J_{2, n}$ is the Jackson kernel

$$
J_{2, n}(x):=\frac{1}{\varkappa_{2, n}}\left(\frac{\sin (n x / 2)}{\sin (x / 2)}\right)^{4}, \quad \varkappa_{2, n}:=\frac{1}{\pi} \int_{-\pi}^{\pi}\left(\frac{\sin (n t / 2)}{\sin (t / 2)}\right)^{4} d t .
$$

It is known that $([15, \mathrm{p} .147])$

$$
\frac{3}{2 \sqrt{2}} n^{3} \leq \varkappa_{2, n} \leq \frac{5}{2 \sqrt{2}} n^{3} .
$$

Jackson kernel $J_{2, n}$ satisfies the relations

$$
\left.\begin{array}{c}
\frac{1}{\pi} \int_{\mathbb{T}} J_{2, n}(u) d u=1, \\
\frac{1}{\pi} \int_{0}^{\pi} u J_{2, n}(u) d u \leq \frac{1}{2 n},
\end{array}\right\}
$$


Lemma 4.1. Let $W(x)=\left|x-x_{0}\right|^{\gamma}, \theta>0, p(.) \in \mathrm{P}_{0}(\mathbb{T})$, and $f \in L_{0, W}^{p(.), \theta}$. If $f \in$ $\mathcal{W}_{0, p(.), W, \theta}^{1}$, then

$$
E_{n}(f)_{p(.), W, \theta} \leq\left\|f-D_{n} f\right\|_{p(.), W, \theta} \leq \frac{c}{n}\left\|f^{\prime}\right\|_{p(.), W, \theta}
$$

holds for $n \in \mathbb{N}$.

Proof of Lemma 4.1. From (4.1), Theorem 2.14, and (4.2), we have

$$
\begin{aligned}
\left\|f-D_{n} f\right\|_{p(.), W, \theta} & =\left\|\frac{1}{\pi} \int_{\mathbb{T}}(f(x)-f(x-t))(1 / t) t J_{2,\left\lfloor\frac{n}{2}\right\rfloor+1}(t) d t\right\|_{p(.), W, \theta} \\
& =\left\|\frac{1}{\pi} \int_{\mathbb{T}} t J_{2,\left\lfloor\frac{n}{2}\right\rfloor+1}(t) \frac{1}{t} \int_{x-t}^{x} f^{\prime}(\tau) d \tau d t\right\|_{p(.), W, \theta} \\
& \leq \frac{1}{\pi} \int_{\mathbb{T}} t J_{2,\left\lfloor\frac{n}{2}\right\rfloor+1}(t)\left\|\frac{1}{t} \int_{x-t}^{x} f^{\prime}(\tau) d \tau\right\|_{p(.), W, \theta} d t \\
& \leq\left\|M f^{\prime}\right\|_{p(.), W, \theta} \frac{1}{\pi} \int_{0}^{\pi} t J_{2,\left\lfloor\frac{n}{2}\right\rfloor+1}(t) d t \\
& \leq \frac{C}{2\left(\left\lfloor\frac{n}{2}\right\rfloor+1\right)}\left\|f^{\prime}\right\|_{p(.), W, \theta} \leq \frac{c}{n}\left\|f^{\prime}\right\|_{p(.), W, \theta} .
\end{aligned}
$$

Hence (4.3) holds.

Proof of Theorem 3.1. Let $f \in \mathcal{W}_{0, p(.), W, \theta}^{1}, n \in \mathbb{N}, \Theta_{n} \in \mathcal{T}_{n}, E_{n}\left(f^{\prime}\right)_{p(.), W, \theta}=\left\|f^{\prime}-\Theta_{n}\right\|_{p(.), W, \theta}$ and $\beta / 2$ be the constant term of $\Theta_{n}$, namely,

$$
\beta=\frac{1}{\pi} \int_{\mathbb{T}} \Theta_{n}(t) d t=\frac{1}{\pi} \int_{\mathbb{T}}\left(\Theta_{n}(t)-f^{\prime}(t)\right) d t
$$

Then

$$
\begin{aligned}
|\beta / 2| & \leq \frac{1}{2 \pi}\left\|f^{\prime}-\Theta_{n}\right\|_{L_{1}} \\
& \leq \frac{c}{2 \pi}\left\|f^{\prime}-\Theta_{n}\right\|_{p(.), W, \theta}=\frac{c}{2 \pi} E_{n}\left(f^{\prime}\right)_{p(.), W, \theta} .
\end{aligned}
$$

On the other hand

$$
\begin{aligned}
\left\|f^{\prime}-\left(\Theta_{n}-\beta / 2\right)\right\|_{p(.), W, \theta} & \leq E_{n}\left(f^{\prime}\right)_{p(.), W, \theta}+\|\beta / 2\|_{p(.), W, \theta} \\
& \leq E_{n}\left(f^{\prime}\right)_{p(.), W, \theta}+\frac{c}{2 \pi}\|W\|_{L_{1}} E_{n}\left(f^{\prime}\right)_{p(.), W, \theta} \\
& =\left(1+\frac{c}{2 \pi}\|W\|_{L_{1}}\right) E_{n}\left(f^{\prime}\right)_{p(.), W, \theta} .
\end{aligned}
$$

Set $u_{n} \in \mathcal{T}_{n}$ so that $u_{n}^{\prime}=\Theta_{n}-\beta / 2$. Then

$$
\begin{aligned}
E_{n}(f)_{p(.), W, \theta} & =E_{n}\left(f-u_{n}\right)_{p(.), W, \theta} \\
& \leq \frac{c}{n}\left\|f^{\prime}-\left(\Theta_{n}-\beta / 2\right)\right\|_{p(.), W, \theta} \\
& \leq\left(c+\frac{C}{2 \pi}\|W\|_{L_{1}}\right) \frac{1}{n} E_{n}\left(f^{\prime}\right)_{p(.), W, \theta}
\end{aligned}
$$

for all $f \in \mathcal{W}_{0, p(.), W, \theta}^{1}$. If $f \in \mathcal{W}_{0, p(.), W, \theta}^{r}$ for some $r$, the last inequality gives

$$
\begin{aligned}
E_{n}(f)_{p(.), W, \theta} & \leq C\left(1+\frac{c}{2 \pi}\|W\|_{L_{1}}\right)^{r} \frac{1}{n^{r}} E_{n}\left(f^{(r)}\right)_{p(.), W, \theta} \\
& =\frac{c}{n^{r}} E_{n}\left(f^{(r)}\right)_{p(.), W, \theta} .
\end{aligned}
$$


Proof of Theorem 3.3. Let $f \in L_{0, W}^{p(.), \theta}$. Using Theorem 3.1 and Corollary 3.2 we have

$$
\begin{aligned}
E_{n}(f)_{p(.), W, \theta} & \leq E_{n}(f-g)_{p(.), W, \theta}+E_{n}(g)_{p(.), W, \theta} \\
& \leq c\left\{\|f-g\|_{p(.), W, \theta}+\delta^{r}\left\|g^{(r)}\right\|_{p(.), W, \theta}\right\} .
\end{aligned}
$$

for $g \in \mathcal{W}_{0, p(.), W, \theta}^{r}$ and $\delta=\frac{1}{n}$. Using Theorem 3.7 and taking infimum on $g \in \mathcal{W}_{0, p(.), W, \theta}^{r}$, we obtain

$$
E_{n}(f)_{p(.), W, \theta} \leq c \Omega_{r}\left(f, \frac{1}{n}\right)_{p(.), W, \theta}, n \in \mathbb{N}
$$

Proof of Theorem 3.4. Let $T_{n}$ be a best approximation trigonmetric polynomial for $f \in L_{0, W}^{p(.), \theta}$. For any $n \in \mathbb{N}$ we choose $n \in \mathbb{N}$ such that $2^{m} \leq n<2^{m+1}$. If we use the subadditivity property of $\Omega_{r}(f, \delta)_{p(.), W, \theta}$, then we have

$$
\Omega_{r}(f, \delta)_{p(.), W, \theta} \leq \Omega_{r}\left(f-T_{2^{m+1}}, \delta\right)_{p(.), W, \theta}+\Omega_{r}\left(T_{2^{m+1}}, \delta\right)_{p(.), W, \theta} \cdot
$$

On the other hand, it is well-known that

$$
2^{(i+1) r} E_{2^{i}}(f)_{p(.), W, \theta} \leq 2^{2 r} \sum_{j=2^{i-1}+1}^{2^{i}} j^{r-1} E_{j}(f)_{p(.), W, \theta}
$$

by Theorem 3.1 in [26]. If we take $\delta=\frac{1}{n}$, then we get

$$
\begin{aligned}
\Omega_{r}\left(f-T_{2^{m+1}}, \delta\right)_{p(.), W, \theta} & \leq c\left\|f-T_{2^{m+1}}\right\|_{p(.), W, \theta} \\
& =c E_{2^{m+1}}(f)_{p(.), W, \theta} \\
& \leq \frac{c}{n^{r}} 2^{2(m+1) r} E_{2^{m}}(f)_{p(.), W, \theta} \\
& \leq c \delta^{r} 2^{2 r} \sum_{k=2^{m-1}+1}^{2^{m}} k^{r-1} E_{k}(f)_{p(.), W, \theta} .
\end{aligned}
$$

Using Lemma 3.5, Lemma 3.8 and (4.5) one can find that

$$
\begin{aligned}
& \Omega_{r}\left(T_{2^{m+1}}, \delta\right)_{p(.), W, \theta} \\
\leq & c \delta^{r}\left\|T_{2^{m+1}}^{(r)}\right\|_{p(.), W, \theta} \\
\leq & c \delta^{r}\left\{\left\|T_{1}^{(r)}+\sum_{i=0}^{m}\left(T_{2^{i+1}}^{(r)}-T_{2^{i}}^{(r)}\right)\right\|_{p(.), W, \theta}\right\} \\
\leq & c \delta^{r}\left\{\left\|T_{1}\right\|_{p(.), W, \theta}+\sum_{i=0}^{m} 2^{(i+1) r}\left\|T_{2^{i+1}}^{(r)}-T_{2^{i}}^{(r)}\right\|_{p(.), W, \theta}\right\} \\
\leq & c \delta^{r}\left\{E_{0}(f)_{p(.), W, \theta}+\sum_{i=0}^{m} 2^{(i+1) r} E_{2^{i}}(f)_{p(.), W, \theta}\right\} \\
= & c \delta^{r}\left\{E_{0}(f)_{p(.), W, \theta}+2^{r} E_{1}(f)_{p(.), W, \theta}+2^{2 r} \sum_{i=1}^{m} \sum_{k=2^{i-1}+1}^{2^{i}} k^{r-1} E_{k}(f)_{p(.), W, \theta}\right\} \\
\leq & c \delta^{r}\left\{E_{0}(f)_{p(.), W, \theta}+\sum_{k=1}^{2^{m}} k^{r-1} E_{k}(f)_{p(.), W, \theta}\right\} .
\end{aligned}
$$


If we combine (4.4), (4.6) and (4.7), then we find

$$
\Omega_{r}\left(f, \frac{1}{n}\right)_{p(.), W, \theta} \leq \frac{c}{n^{r}} \sum_{k=0}^{n}(k+1)^{r-1} E_{k}(f)_{p(.), W, \theta}, n \in \mathbb{N} .
$$

The notation $\mathcal{O}$ indicates that $A=\mathcal{O}(B)$ if and only if there exists a positive constant $c$, independent of essential parameters, such that $A \leq c B$.

Corollary 4.2. If $E_{n}(f)_{p(.), W, \theta}=\mathcal{O}\left(n^{-\alpha}\right), \alpha>0$, then under the conditions of Theorem 3.4 we have

$$
\Omega_{r}(f, \delta)_{p(.), W, \theta}= \begin{cases}\mathcal{O}\left(\delta^{\alpha}\right) & , r>\alpha, \\ \mathcal{O}\left(\delta^{\alpha} \log \left(\frac{1}{\delta}\right)\right) & , r=\alpha, \\ \mathcal{O}\left(\delta^{r}\right) & , r<\alpha .\end{cases}
$$

Definition 4.3. Let $W(x)=\left|x-x_{0}\right|^{\gamma}, \theta>0, p(.) \in P_{0}(\mathbb{T}), f \in L_{0, W}^{p(.), \theta}, \alpha>0$ and $r:=[\alpha]+1([\alpha]$ is the integer part of $\alpha)$. We define the generalized Lipschitz class as

$$
\operatorname{Lip}_{p(.), W, \theta}^{\alpha, r}=\left\{f \in L_{W}^{p(.), \theta}: \Omega_{r}(f, \delta)_{p(.), W, \theta}=\mathcal{O}\left(\delta^{\alpha}\right)\right\} .
$$

Corollary 4.4. Let $W(x)=\left|x-x_{0}\right|^{\gamma}, \theta>0, p(.) \in \mathrm{P}_{0}(\mathbb{T}), f \in L_{0, W}^{p(.), \theta}$ and $\alpha>0$. Then the following statements are equivalent:

(i) $f \in \operatorname{Lip}_{p(.), W, \theta}^{\alpha, r}$

(ii) $E_{n}(f)_{p(.), W, \theta}=O\left(n^{-\alpha}\right), n \in \mathbb{N}$.

Theorem 4.5. Let $W(x)=\left|x-x_{0}\right|^{\gamma}, \theta>0, p(.) \in \mathrm{P}_{0}(\mathbb{T}), f \in L_{0, W}^{p(.), \theta}$ and $r \in \mathbb{N}$. If

$$
\sum_{k=1}^{\infty} k^{r-1} E_{k}(f)_{p(.), W, \theta}<\infty
$$

then, $f \in \mathcal{W}_{p(.), 0, W, \theta}^{r}$ and

$$
E_{n}\left(f^{(r)}\right)_{p(.), W, \theta} \leq c\left(n^{r} E_{n}(f)_{p(.), W, \theta}+\sum_{k=n+1}^{\infty} k^{r-1} E_{k}(f)_{p(.), W, \theta}\right)
$$

with a positive constant $c$ independent of $f$ and $n$.

Proof of Theorem 4.5. For the polynomial $T_{n}$ of the best approximation to $f$ we have by Lemma 3.8 that

$$
\begin{aligned}
\left\|T_{2^{i+1}}^{(r)}-T_{2^{i}}^{(r)}\right\|_{p(.), W, \theta} & \leq C(r) 2^{(i+1) r}\left\|T_{2^{i+1}}-T_{2^{i}}\right\|_{p(.), W, \theta} \\
& \leq 2 C(r) 2^{(i+1) r} E_{2^{i}}(f)_{p(.), W, \theta} .
\end{aligned}
$$

Hence

$$
\begin{aligned}
\sum_{i=1}^{\infty}\left\|T_{2^{i+1}}-T_{2^{i}}\right\|_{p(.), W, \theta}^{r} & =\sum_{i=1}^{\infty}\left\|T_{2^{i+1}}^{(r)}-T_{2^{i}}^{(r)}\right\|_{p(.), W, \theta}+\sum_{i=1}^{\infty}\left\|T_{2^{i+1}}-T_{2^{i}}\right\|_{p(.), W, \theta} \\
& \leq c \sum_{m=2}^{\infty} m^{r-1} E_{m}(f)_{p(.), W, \theta}<\infty .
\end{aligned}
$$

Therefore

$$
\left\|T_{2^{i+1}}-T_{2^{i}}\right\|_{p(.), W, \theta}^{r} \rightarrow 0 \text { as } i \rightarrow \infty .
$$

This means that $\left\{T_{2^{i}}\right\}$ is a Cauchy sequence in $L_{W}^{p(.), \theta}$. Since $T_{2^{i}} \rightarrow f$ in $L_{W}^{p(.), \theta}$ and $\mathcal{W}_{p(.), W, \theta}^{r}$ is a Banach space we obtain $f \in \mathcal{W}_{p(.), W, \theta}^{r}$. 
On the other hand since

$$
\left\|f^{(r)}-T_{n}^{(r)}\right\|_{p(.), W, \theta} \leq\left\|T_{2^{m+2}}^{(r)}-T_{n}^{(r)}\right\|_{p(.), W, \theta}+\sum_{k=m+2}^{\infty}\left\|T_{2^{k+1}}^{(r)}-T_{2^{k}}^{(r)}\right\|_{p(.), W, \theta}
$$

for $2^{m} \leq n<2^{m+1}$, we have

$$
\left\|T_{2^{m+2}}^{(r)}-T_{n}^{(r)}\right\|_{p(.), W, \theta} \leq c 2^{(m+2) r} E_{n}(f)_{p(.), W, \theta} \leq c(n+1)^{r} E_{n}(f)_{p(.), W, \theta} .
$$

Also we find

$$
\begin{aligned}
\sum_{k=m+2}^{\infty}\left\|T_{2^{k+1}}^{(r)}-T_{2^{k}}^{(r)}\right\|_{p(.), W, \theta} & \leq c \sum_{k=m+2}^{\infty} 2^{(k+1) r} E_{2^{k}}(f)_{p(.), W, \theta} \\
& \leq c \sum_{k=m+2}^{\infty} \sum_{\mu=2^{k-1}+1}^{2^{k}} \mu^{r-1} E_{\mu}(f)_{p(.), W, \theta} \\
& =c \sum_{\nu=2^{m+1}+1}^{\infty} \nu^{r-1} E_{\nu}(f)_{p(.), W, \theta} \\
& \leq c \sum_{\nu=n+1}^{\infty} \nu^{r-1} E_{\nu}(f)_{p(.), W, \theta} .
\end{aligned}
$$

This completes the proof.

A polynomial $T \in \Pi_{n}$ is said to be a near best approximant of $f \in L_{0, W}^{p(.), \theta}$ for $W(x)=$ $\left|x-x_{0}\right|^{\gamma}, \theta>0, p(.) \in P_{0}(\mathbb{T})$, if

$$
\|f-T\|_{p(.), W, \theta} \leq c E_{n}(f)_{p(.), W, \theta}, \quad n=1,2, \ldots .
$$

Theorem 4.6. Let $W(x)=\left|x-x_{0}\right|^{\gamma}, \theta>0, p(.) \in \mathrm{P}_{0}(\mathbb{T}), r, n \in \mathbb{N}$. If $T_{n} \in \Pi_{n}$ is a near best approximant of $f \in \mathcal{W}_{p(.), W, \theta}^{r}$, then there exists a constant $c>0$ dependent only on $r, W$ and $p($.$) , such that$

$$
\left\|f^{(r)}-T_{n}^{(r)}\right\|_{p(.), W, \theta} \leq c E_{n}\left(f^{(r)}\right)_{p(.), W, \theta} .
$$

Corollary 4.7. Suppose that $W(x)=\left|x-x_{0}\right|^{\gamma}, \theta>0, p(.) \in \mathrm{P}_{0}(\mathbb{T}), r, n \in \mathbb{N}, f \in$ $\mathcal{W}_{p(.), W, \theta}^{\alpha}$, and

$$
\sum_{\nu=1}^{\infty} \nu^{\alpha-1} E_{\nu}(f)_{p(.), W, \theta}<\infty
$$

for some $\alpha>0$. Hence there exists a constant $c>0$ dependent only on $\alpha, r, W$ and $p($. such that

$$
\left.\left.\Omega_{r}\left(f^{(\alpha)}, \frac{\pi}{n}\right)_{p(.), W, \theta} \leq c\left\{\frac{1}{n^{r}} \sum_{\nu=0}^{n}(\nu+1)^{\alpha+r-1} E_{\nu}(f)_{p(.), W, \theta}+\sum_{\nu=n+1}^{\infty} \nu^{\alpha-1} E_{\nu}\right) f\right)_{p(.), W, \theta}\right\} .
$$

Proof of Theorem 4.6. We set $W_{n}(f):=W_{n}(x, f):=\frac{1}{n+1} \sum_{\nu=n}^{2 n} S_{\nu}(x, f), \quad n=0,1,2, \ldots$. Since

then we have

$$
W_{n}\left(., f^{(\alpha)}\right)=W_{n}^{(\alpha)}(., f)
$$

$$
\begin{gathered}
\left\|f^{(\alpha)}(.)-T_{n}^{(\alpha)}(., f)\right\|_{p(.), W, \theta} \leq\left\|f^{(\alpha)}(.)-W_{n}\left(., f^{(\alpha)}\right)\right\|_{p(.), W, \theta} \\
+\left\|T_{n}^{(\alpha)}\left(., W_{n}(f)\right)-T_{n}^{(\alpha)}(., f)\right\|_{p(.), W, \theta}+\left\|W_{n}^{(\alpha)}(., f)-T_{n}^{(\alpha)}\left(., W_{n}(f)\right)\right\|_{p(.), W, \theta} \\
=I_{1}+I_{2}+I_{3} .
\end{gathered}
$$


We denote by $T_{n}^{*}(x, f)$ the best approximating polynomial of degree at most $n$ to $f$ in $L_{W}^{p(.), \theta}$. In this case, from the boundedness of $W_{n}$ in $L_{W}^{p(.), \theta}$, we have

$$
\begin{aligned}
I_{1} & \leq\left\|f^{(\alpha)}(.)-T_{n}^{*}\left(., f^{(\alpha)}\right)\right\|_{p(.), W, \theta}+\left\|T_{n}^{*}\left(., f^{(\alpha)}\right)-W_{n}\left(., f^{(\alpha)}\right)\right\|_{p(.), W, \theta} \\
& \leq c(p, W, \theta) E_{n}\left(f^{(\alpha)}\right)_{p(.), W, \theta}+\left\|W_{n}\left(., T_{n}^{*}\left(f^{(\alpha)}\right)-f^{(\alpha)}\right)\right\|_{p(.), W, \theta} \\
& \leq c(p, W, \theta) E_{n}\left(f^{(\alpha)}\right)_{p(.), W, \theta} .
\end{aligned}
$$

From Lemma 3.8 we get

$$
I_{2} \leq c(p, W, \theta) n^{\alpha}\left\|T_{n}\left(., W_{n}(f)\right)-T_{n}(., f)\right\|_{p(.), W, \theta}
$$

and

$$
\begin{aligned}
I_{3} & \leq c(p, W, \theta)(2 n)^{\alpha}\left\|W_{n}(., f)-T_{n}\left(., W_{n}(f)\right)\right\|_{p(.), W, \theta} \\
& \leq c(p, W, \theta)(2 n)^{\alpha} E_{n}\left(W_{n}(f)\right)_{p(.), W, \theta} .
\end{aligned}
$$

Now we have

$$
\begin{aligned}
\left\|T_{n}\left(., W_{n}(f)\right)-T_{n}(., f)\right\|_{p(.), W, \theta} \leq\left\|T_{n}\left(., W_{n}(f)\right)-W_{n}(., f)\right\|_{p(.), W, \theta} \\
\quad+\left\|W_{n}(., f)-f(.)\right\|_{p(.), W, \theta}+\left\|f(.)-T_{n}(., f)\right\|_{p(.), W, \theta} \\
\leq \quad c(p, W, \theta) E_{n}\left(W_{n}(f)\right)_{p(.), W, \theta}+c(p, W, \theta) E_{n}(f)_{p(.), W, \theta} \\
\quad+c(p, W, \theta) E_{n}(f)_{p(.), W, \theta} .
\end{aligned}
$$

Since

$$
E_{n}\left(W_{n}(f)\right)_{p(.), W, \theta} \leq c(p, W, \theta) E_{n}(f)_{p(.), W, \theta}
$$

then we get

$$
\begin{aligned}
\left\|f^{(\alpha)}(.)-T_{n}^{(\alpha)}(., f)\right\|_{p(.), W, \theta} \leq & c(p, W, \theta) E_{n}\left(f^{(\alpha)}\right)_{p(.), W, \theta} \\
& +c(p, W, \theta) n^{\alpha} E_{n}\left(W_{n}(f)\right)_{p(.), W, \theta} \\
+ & c(p, W, \theta) n^{\alpha} E_{n}(f)_{p(.), W, \theta}+c(p, W, \theta)(2 n)^{\alpha} E_{n}\left(W_{n}(f)\right)_{p(.), W, \theta} \\
\leq \quad & c(p, W, \theta) E_{n}\left(f^{(\alpha)}\right)_{p(.), W, \theta}+c(p, W, \theta) n^{\alpha} E_{n}(f)_{p(.), W, \theta} .
\end{aligned}
$$

Since, according to Theorem 3.1,

$$
E_{n}(f)_{p(.), W, \theta} \leq \frac{c(p, W, \theta)}{(n+1)^{\alpha}} E_{n}\left(f^{(\alpha)}\right)_{p(.), W, \theta},
$$

we obtain

$$
\left\|f^{(\alpha)}(.)-T_{n}^{(\alpha)}(., f)\right\|_{p(.), W, \theta} \leq c(p, W, \theta) E_{n}\left(f^{(\alpha)}\right)_{p(.), W, \theta}
$$

and the proof is completed.

Acknowledgment. The authors would like to thank the referee for the helpful comments and suggestions. 


\section{References}

[1] G. Anatriello, Iterated grand and small Lebesgue spaces, Collect. Math. 65, 273-284, 2014.

[2] R. Akgün, Trigonometric approximation of functions in generalized Lebesgue spaces with variable exponent, Ukrainian Math. J. 63 (1), 1-26, 2011.

[3] R. Akgün, Approximating polynomials for functions of weighted Smirnov-Orlicz spaces, J. Funct. Spaces Appl. 2012, Art. ID 982360, 2012.

[4] R. Akgün and D.M. Israfilov, Approximation and moduli of smoothness of fractional order in Smirnov-Orlicz spaces, Glas. Mat. Ser. III, 42 (2), 121-136, 2008.

[5] R. Akgün and D.M. Israfilov, Polynomial approximation in weighted Smirnov Orlicz space, Proc. A. Razmadze Math. Inst. 139, 89-92, 2005.

[6] R. Akgün and D.M. Israfilov, Approximation by interpolating polynomials in SmirnovOrlicz class, J. Korean Math. Soc. 43 (2), 413-424, 2006.

[7] R. Akgün and D.M. Israfilov, Simultaneous and converse approximation theorems in weighted Orlicz spaces, Bull. Belg. Math. Soc. 17 (2), 13-28, 2010.

[8] R. Akgün and V. Kokilashvili, On converse theorems of trigonometric approximation in weighted variable exponent Lebesgue spaces, Banach J. Math. Anal. 5 (1), 70-82, 2011.

[9] I. Aydin, Weighted variable Sobolev spaces and capacity, J. Funct. Spaces Appl. 2012, Art. ID 132690, 2012.

[10] D. Cruz-Uribe and A. Fiorenza, Variable Lebesgue Spaces: Foundations and Harmonic Analysis (Applied and Numerical Harmonic Analysis), Birkhäuser/Springer, Heidelberg, 2013.

[11] D. Cruz-Uribe, L. Diening and P. Hästö, The maximal operator on weighted variable Lebesgue spaces, Fract. Calc. Appl. Anal. 14 (3), 361-374, 2011.

[12] N. Danelia and V. Kokilashvili, Approximation by trigonometric polynomials in the framework of variable exponent Lebesgue spaces, Georgian Math. J. 23 (1), 43-53, 2016.

[13] L. Diening, Maximal function on generalized Lebesgue spaces $L^{p(.)}$, Math. Inequal. Appl. 7, 245-253, 2004.

[14] L. Diening, P. Harjulehto, P. Hästö and M. Ruzicka, Lebesgue and Sobolev Spaces with Variable Exponents, Lecture Notes in Mathematics, 2017, Springer, Heidelberg, 2011.

[15] V.K. Dzyadyk and I.A. Shevchuk, Theory of Uniform Approximation of Functions by Polynomials, Walter de Gruyter GmbH \& Co. KG, 10785 Berlin, Germany, 2008.

[16] A. Fiorenza, B. Gupta and P. Jain, The maximal theorem in weighted grand Lebesgue spaces, Studia Math. 188 (2), 123-133, 2008.

[17] A. Fiorenza, V. Kokilashvili and A. Meskhi, Hardy-Littlewood maximal operator in weighted grand variable exponent Lebesgue space, Mediterr. J. Math. 14 (118), 2017.

[18] L. Greco, T. Iwaniec and C. Sbordone, Inverting the p-harmonic operator, Manuscripta Math. 92, 249-258, 1997.

[19] D.M. Israfilov and R. Akgün, Approximation in weighted Smirnov-Orlicz classes, J. Math. Kyoto Univ. 46 (4), 755-770, 2006.

[20] D.M. Israfilov and A. Testici, Approximation in weighted generalized grand Lebesgue spaces, Colloq. Math. 143, 113-126, 2016.

[21] D.M. Israfilov and A. Testici, Approximation in weighted generalized grand Smirnov spaces, Studia Sci. Math. Hungar. 54 (4), 471-488, 2017.

[22] T. Iwaniec and C. Sbordone, On integrability of the Jacobien under minimal hypotheses, Arch. Ration. Mech. Anal. 119, 129-143, 1992.

[23] V. Kokilashvili and A. Meskhi, Maximal and Calderon-Zygmund operators in grand variable exponent Lebesgue spaces, Georgian Math. J. 21, 447-461, 2014. 
[24] O. Kováčik and J. Rákosník, On spaces $L^{p(x)}$ and $W^{k, p(x)}$, Czechoslovak Math. J. 41 (116), 592-618, 1991.

[25] W. Orlicz. Über konjugierte exponentenfolgen, Stud. Math. 3, 200-211, 1931.

[26] R.A. De Vore and G.G. Lorentz, Constructive Approximation, Springer, 1993.

[27] A. Zygmund, Trigonometric Series, Volume I-II, Cambridge University Press, Cambridge, 1968. 